\title{
Biosynthesis of silver nanoparticles from Cavendish banana peel extract and its antibacterial and free radical scavenging assay: a novel biological approach
}

\author{
T. Kokila • P. S. Ramesh • D. Geetha
}

Received: 17 November 2014/ Accepted: 12 January 2015/Published online: 29 January 2015

(C) The Author(s) 2015. This article is published with open access at Springerlink.com

\begin{abstract}
Biosynthesis of metallic silver nanoparticles has now become an alternative to physical and chemical approaches. In the present study, silver nanoparticles (AgNPs) were synthesized from Cavendish banana peel extract (CBPE) and characterized by UV-visible spectroscopy, X-ray diffraction (XRD), Fourier transform infrared (FT-IR) spectroscopy, Atomic force microscopy (AFM), Field emission scanning electronic microscope (FESEM), Dynamic light scattering (DLS) and zeta potential (ZP). The AgNPs formation was confirmed by UV-visible spectroscopy through color conversion due to surface plasma resonance band at $430 \mathrm{~nm}$. The effect of $\mathrm{pH}$ on nanoparticle synthesis was determined by adjusting the various $\mathrm{pH}$ of the reaction mixtures. The crystalline nature of nanoparticles was confirmed from the XRD pattern, and the grain size was found to be around $34 \mathrm{~nm}$. To identify the compounds responsible for the bioreduction of $\mathrm{Ag}^{+}$ion and the stabilization of AgNPs produced, the functional group present in Cavendish banana peel extract was investigated using FTIR. AFM has proved to be very helpful in determining morphological features and the diameter of AgNPs in the range of $23-30 \mathrm{~nm}$ was confirmed by FESEM. DLS studies revealed that the average size of AgNPs was found to be around $297 \mathrm{~nm}$. Zeta potential value for AgNPs obtained was $-11 \mathrm{mV}$ indicating the moderate stability of synthesized nanoparticles. The antibacterial activity of the nanoparticles was studied
\end{abstract}

\section{P. S. Ramesh ( $\square)$}

Physics Wing DDE, Annamalai University, Annamalai Nagar, Chidambaram 608002, TamilNadu, India

e-mail: psrddephyau@gmail.com

T. Kokila $\cdot$ D. Geetha

Department of Physics, Annamalai University, Annamalai

Nagar, Chidambaram 608002, TamilNadu, India against Gram-positive and Gram-negative bacteria. Biosynthesized AgNPs showed a strong DPPH radical and ABTS scavengers compared to the aqueous peel extract of Cavendish banana.

Keywords Silver nanoparticles - Biosynthesis · Cavendish banana peel $\cdot$ Antibacterial $\cdot$ Free radical scavenging

\section{Introduction}

Nanotechnology is emerging as a rapidly growing field with its application in science and technology (Albrecht et al. 2006). Nanobiotechnology is a field that interrelates both biological sciences and nanotechnology. It provides a platform for the development of ecofriendly and the green synthesis of nanoparticles with the help of biological sources like plants and microorganisms (Guangquan et al. 2012). Nanoparticles exhibit completely new or improved properties based on specific characteristics, such as size, distribution and morphology.

Metal nanoparticles have been of great interest due to their distinctive features such as catalytic, optical, magnetic, and electrical properties. Nanoparticles of noble metals, such as silver, gold and platinum, are widely applied in products that directly come in contact with the human body, such as shampoos, soaps, detergent, shoes, cosmetic products, and toothpaste, besides medical and pharmaceutical applications (Gong et al. 2007). The silver nanoparticles have various important applications. Historically, silver has been known to have a disinfecting effect and has been found in applications ranging from traditional medicines to culinary items. It has been reported that silver nanoparticles are non-toxic to humans and most effective 
against bacteria, virus and other eukaryotic microorganisms at low concentrations and without any side effects (Jeong et al. 2005).

A number of approaches are available for the synthesis of silver nanoparticles: Facile method (Nidhi et al. 2009), thermal decomposition of silver compounds (Navaladian et al. 2007), electrochemical (Starowicz et al. 2006), sonochemical (Easu et al. 2010), microwave-assisted process (Sreeram et al. 2008) and recently via green chemistry route (Begum et al. 2009). Unfortunately, many of the nanoparticle synthesis or production methods involve the use of hazardous chemicals, low material conversions, high energy requirements, difficult and wasteful purifications. Therefore, there is a growing need to develop environmentally friendly processes for nanoparticle synthesis without using toxic chemicals. Biosynthetic methods employing either microorganisms or plant extracts have emerged as a simple and viable alternative to chemical synthetic procedures and physical methods (Shanmugavadivu et al. 2014).

Green nanoparticle synthesis has been achieved using environmentally acceptable plant extract and ecofriendly reducing and capping agent. Many reports are available on biosynthesis of silver nanoparticles by plant parts like Punica granatum peels (Ahmad et al. 2012), Citrus sinensis peel (Kaviya et al. 2011), lemon leaves (Padma and Shukla 2012), Myrica esculenta leaf (Phanjom et al. 2012), Wrightia tinctoria leaves (Bharani et al. 2012) and Mango peel (Yang and Li 2013). In this work, a novel biological route for the synthesis of silver nanoparticles using Cavendish banana peel extract is demonstrated.

The peels of a variety of fruits have gained attention as a natural source of antioxidants and phytochemical content which are rich in compounds with free radical scavenging activity. Banana and Plantain peels are major agricultural wastes which have been used as medicine, animal feeds, blacking of leathers, soap making, fillers in rubber and so on (Arawande and Komolafe 2010). Banana peel, an underutilized source of phenolic compounds, is considered a good source of antioxidants for foods and functional foods against cancer and heart disease (Someya et al. 2002). The peel of the fruit contains various antioxidant compounds, such as gallocatechin (Someya et al. 2002) and dopamine (Kanazawa and Sakakibara 2000). A few applications of banana peels discussed in the literature include (1) exploitation for their medicinal properties (Parmar and Kar 2008), (2) in ethanol fermentation (Tewari et al. 1986), (3) application as a substrate for generating fungal biomass (Essien et al. 2005), (4) use in the production of laccase (Osma et al. 2007) and (5) utilization as a biosorbent for heavy metal removal (Annadurai et al. 2003). On the basis of the available literature, we hypothesized that banana peels that are inherently rich in polymers such as lignin, hemicellulose and pectins (Happi Emaga et al. 2007) could be used in the synthesis of silver nanoparticles.

\section{Materials and methods}

\section{Materials}

The Banana fruit was collected from Agricultural farm, Annamalai University. Silver nitrate was purchased from Sigma-Aldrich and used as received. Double distilled water was used for the experiments. All glass wares were properly washed with distilled water and dried in oven.

\section{Preparation of CBP extract}

Banana peel was used as a reducing agent for the development of silver nanoparticles. The fresh banana was washed repeatedly with distilled water to remove the dust and organic impurities present in it. Then, the peels were removed and dried on paper toweling. About $25 \mathrm{~g}$ of peel was taken in a $100 \mathrm{ml}$ beaker containing $50 \mathrm{ml}$ double distilled water and then the peel was boiled at 80 for $10 \mathrm{~min}$ and filtered through Whatman No. 1 filter paper twice. The resultant filtrate was stored at $4{ }^{\circ} \mathrm{C}$ and used as reducing and stabilizing agent.

\section{Synthesis of silver nanoparticles}

To optimize the synthesis route for producing the AgNPs, peel extract concentrations were varied on precursor solution. The various concentrations $(1-5 \mathrm{ml})$ of aqueous peel extract were added to the $100 \mathrm{ml}$ aqueous solution of $1 \mathrm{mM} \mathrm{AgNO}$. The reduction of silver ions takes place within $30 \mathrm{~min}$ at room temperature. The color change of the solution viz., brownish-orange color was observed, indicating the formation of silver nanoparticles.

\section{Characterization of silver nanoparticles}

The nanoparticles were primarily characterized by UVVisible spectroscopy, which has proved to be a very useful technique for the analysis of nanoparticles. UltravioletVisible spectra were obtained using a Shimadzu UV1650pc Spectrophotometer. XRD analysis was carried out on an X-Ray diffractometer (X'Pert-PRO). The high resolution on XRD patterns measured at $3 \mathrm{KW}$ with $\mathrm{Cu}$ target using a scintillation counter $(\lambda=1.5406 \AA)$ at $40 \mathrm{kV}$ and $40 \mathrm{~mA}$ was recorded in the range of $2 \theta=10^{\circ}-80^{\circ}$. The changes in the surface chemical bondings and surface composition were characterized using Fourier Transform Infrared (FT-IR) spectroscopy (Nicolet Avatar series 330) ranging from 400 to $4,000 \mathrm{~cm}^{-1}$. The size and morphology 
of AgNPs were performed by Atomic force microscopy (AGILENT-N9410A series 5500) and JSM-6701F field emission scanning electron microscope (FESEM).The average size of AgNPs in aqueous medium was used to determine hydrodynamic diameter by DLS. The Zeta potential was measured with a Zeta sizer Nano ZS90 (Melvern International Ltd.) Instrument.

\section{Antibacterial activity}

The antibacterial potential of biosynthesized AgNPs and CBPE was tested against Gram-positive (Staphylococcus aureus and Bacillus subtilis) and Gram-negative (Escherichia coli and Klebsiella pneumonia) bacteria using agar disc diffusion method. Approximately, $20 \mathrm{ml}$ of molten and cooled nutrient agar media was poured into sterilized petridishes. The plates were left overnight at room temperature to allow any contamination to appear. The discs were placed on Muller Hinton agar plates inoculated with each of the previously mentioned microorganisms. $25 \mu \mathrm{L}$ of ciprofloxacin was used as positive control (PC). The test plates were incubated at $37^{\circ} \mathrm{C}$ for $24 \mathrm{~h}$. After the incubation period, the zone of inhibition (in $\mathrm{mm}$ diameter) was observed and tabulated.

Free radical scavenging activity

(1) DPPH (1,1-Diphenyl-2-Picryl Hydroxyl) radical scavenging assay: DPPH scavenging activity was measured by slightly modified spectrophotometric method of Brand-Williams et al. (1995). DPPH is a stable free radical and accepts an electron, or hydrogen radical to become a stable diamagnetic molecule. DPPH reacts with an antioxidant compound that can donate hydrogen and get reduced. The change in color (from deep violet to blue) was measured. The intensity of the yellow color developed depends on the amount and nature of radical scavenger present in the sample.

\section{Procedure}

Different concentrations $(50,100,150,200,250 \mu \mathrm{l})$ of CBPE and biosynthesized AgNPs were added, in equal volume, to $0.1 \mathrm{mM}$ methanolic DPPH solution. The reaction mixture was incubated for $30 \mathrm{~min}$ at room temperature under shaking condition and the absorbance was recorded at $517 \mathrm{~nm}$. BHT was used as a standard.
$\%$ scavenging activity

$$
=\frac{\text { Absorbance of control }- \text { Absorbance of sample }}{\text { Absorbance of control }} \times 100
$$

(2) ABTS [2,2'-Azinobis-(3-Ethylbenzothiazoline-6-Sulfonic Acid)] Assay: the total antioxidant activity of the samples was measured by [2,2'-azino-bis (3ethylbenzthiazoline-6-sulphonic acid)] ABTS radical cation decolorization assay according to the method of Re et al. (1999).

\section{Procedure}

ABTS was produced by reacting $7 \mathrm{mM}$ ABTS aqueous solution with $2.4 \mathrm{mM}$ potassium persulfate in the dark for $12-16 \mathrm{~h}$ at room temperature. The radical was stable in this form for more than 2 days when stored in the dark at room temperature. Then, $2 \mathrm{ml}$ of diluted ABTS solution was added to the sample in varying concentrations of CBPE. The blank contained water in place of peel extract. After 30 min of incubation at room temperature, the absorbance was recorded at $734 \mathrm{~nm}$ and compared with standard BHT (Butylated hydroxytoluene). Percentage of inhibition was calculated.

$\%$ scavenging $=\frac{\text { Control OD }- \text { Test OD }}{\text { Control }} \times 100$

OD-Optical Density.

\section{Results and discussion}

Biosynthesis of silver nanoparticles and its mechanism

The detailed study of the biosynthesis of silver nanoparticles was performed using Cavendish banana peel extract. The formation of AgNPs of the filtrate was investigated by the observation of the change in the color of the solution. The appearance of a brownish-orange color in the reaction vessels suggested the formation of AgNPs. Figure 1 shows the photograph of the color change of the AgNPs: the first vial contains Silver nitrate, the second vial contains Cavendish banana peel extract and the third contains AgNPs in colloidal form. Banana peels are mainly composed of pectin, cellulose and hemicelluloses and the functional groups associated with these polymers as well as the proteinaceous matter may be involved in reducing the silver salt to $\mathrm{Ag}^{0}$. Biological components are known to interact 

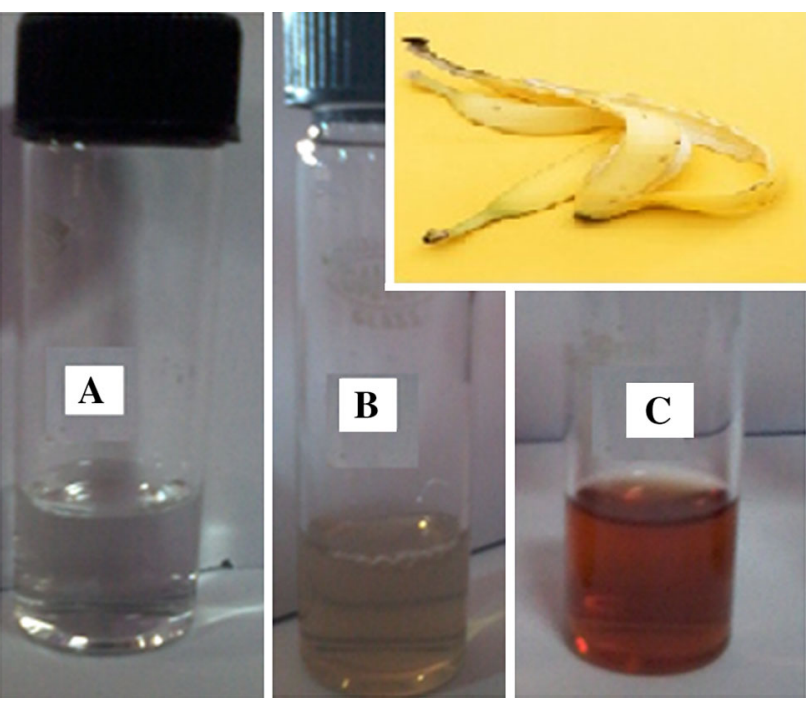

Fig. 1 Vials containing samples of a silver nitrate, b Cavendish banana peel extract, $\mathbf{c}$ colloidal AgNPs by bioreduction of CBPE

with metal salts via these functional groups and mediate their reduction to nanoparticles (Bar et al. 2009; Ganesh Babu and Gunasekaran 2009).

\section{UV-Visible analysis}

The preliminary detection of AgNPs was carried out by visual observation of the color changes of the reaction solutions. These changes were attributed to the excitation of surface plasmon resonance (SPR) in the metal nanoparticles. Typically, UV-visible absorption is used to investigate SPR. Characteristic surface plasmon absorption band was observed at $430 \mathrm{~nm}$ for the brownish-orange colored silver nanoparticles synthesized from $1 \mathrm{mM}$ silver nitrate with peel extract concentration (1-5 ml). It has been found that the optimum concentration for the synthesis of AgNPs is $4 \mathrm{ml}$ extract of $\mathrm{Ag}^{+}$ions. There is small increase in the intensity of SPR band from 1 to $4 \mathrm{ml}$. However, when the concentration is increased further, there is a decrease in the intensity of SPR band. The increase in SPR band intensity [Fig. $2 \mathrm{i}(\mathrm{a}-\mathrm{d})]$ is due to the formation of more AgNPs because of high initial concentration of $\mathrm{Ag}^{+}$ ions. The regular decrease in SPR band intensity from curve (d-e) supports the formation of large sized AgNPs. The AgNPs prepared from $4 \mathrm{ml}$ concentration of $\mathrm{Ag}^{+}$are used for other characterizations. According to Mie's theory, only a single SPR band is expected in the absorption spectra of spherical metal nanoparticles, whereas anisotropic particles could give rise to two or more SPR bands, depending on the shape of the particles ( $\mathrm{He}$ et al. 2002; Novak and Feldheim 2000). In our case, a single SPR peak was observed, which suggests that our AgNPs were spherical in shape.

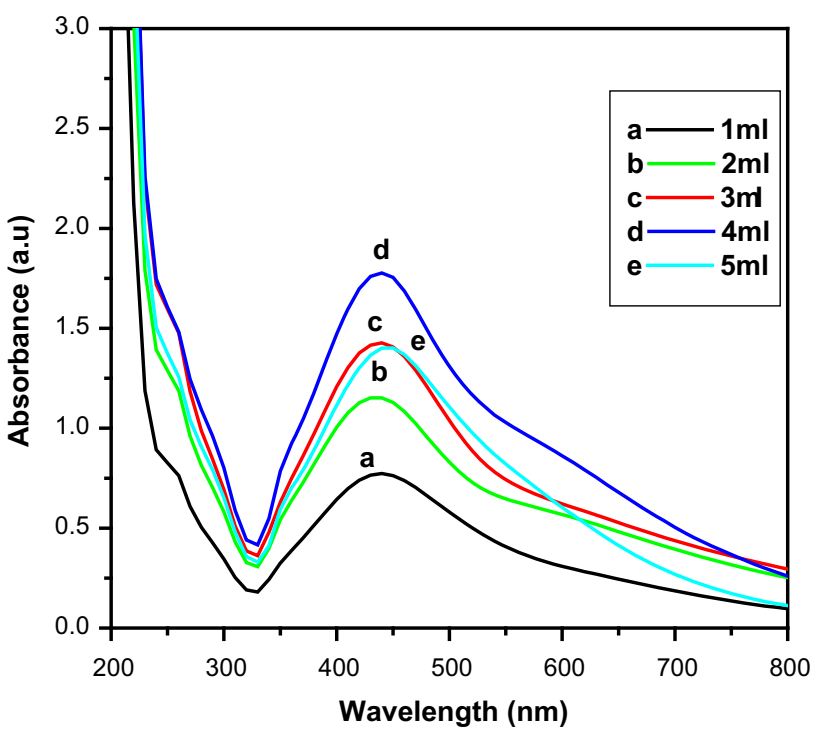

(i)

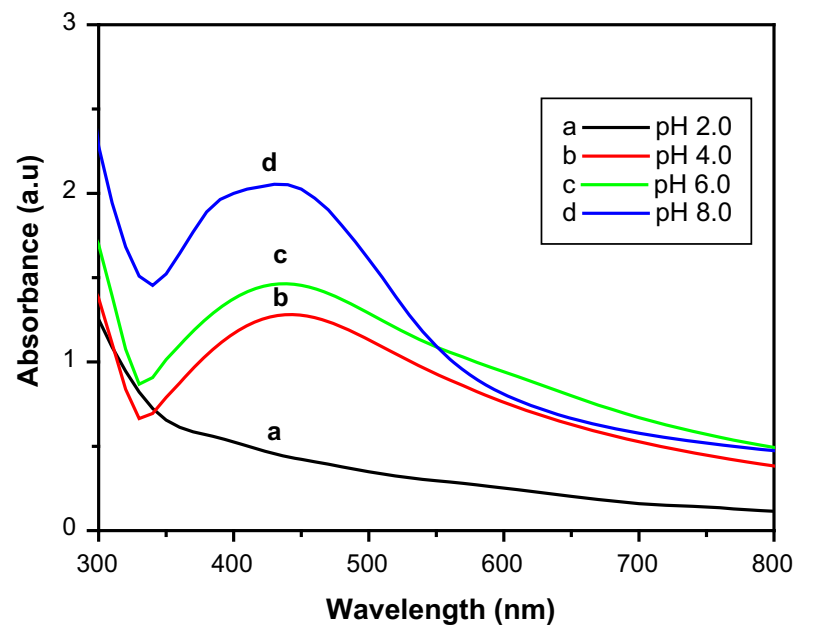

(ii)

Fig. 2 i UV Vis spectra for the AgNPs prepared with $1 \mathrm{mM}$ aqueous $\mathrm{AgNO}_{3}$ solution with various CBPE concentration $(a 1 \mathrm{ml}, b 2 \mathrm{ml}$, $c 3 \mathrm{ml}, d 4 \mathrm{ml}$ and $e 5 \mathrm{ml}$ ). ii UV Vis spectra for the AgNPs prepared with $1 \mathrm{mM}$ aqueous $\mathrm{AgNO}_{3}$ solution of $4 \mathrm{ml} \mathrm{CBPE}$ at different $\mathrm{pH}$ values $(a 2 \mathrm{pH}, b 4 \mathrm{pH}, c 6 \mathrm{pH}$ and $d 8 \mathrm{pH})$

The effect of $\mathrm{pH}$ on the formation of AgNPs was evaluated by UV-visible spectroscopic studies and is given in Fig. 2ii. From the figure, it is evident that the formation of AgNPs mainly depends on the $\mathrm{pH}$ of the reaction medium. The absorbance value was increased gradually with increasing $\mathrm{pH}$ range from 2 to 8 , suggesting that the rate of formation of AgNPs is high in basic $\mathrm{pH}$ than in acidic $\mathrm{pH}$. The formation of AgNPs occurs rapidly, in neutral, and the basic $\mathrm{pH}$ may be due to the ionization of the phenolic group present in the extract (Martinez-Castanon et al. 2008). The slow rate of formation and aggregation of AgNPs at acidic $\mathrm{pH}$ could be related to electrostatic repulsion of anions 
present in the solution (Sun et al. 2008). At basic $\mathrm{pH}$ range, there is a possibility of $\mathrm{Ag}^{+}$precipitating as $\mathrm{AgOH}$.

X-ray diffraction analysis (XRD)

The crystalline nature of AgNPs was further confirmed by $\mathrm{X}$-ray diffraction (XRD) analysis. X-ray diffraction is used to characterize crystallographic structure, grain size, and preferred orientation in polycrystalline or powder solid samples. The XRD patterns of synthesized AgNPs are shown in Fig. 3. Silver nanoparticles synthesized from Banana peel extract showed Bragg Reflection peaks at $38.06^{\circ}, 44.26^{\circ}, 64.5^{\circ}$ and $77.30^{\circ}$ in the $2 \theta$ range between $10-80^{\circ}$ which can be indexed to the (111), (200), (220) and (311) planes of face centered cubic (fcc) crystal, respectively. They have a good match with the standard diffraction pattern of JCPDS No. 89-3722, revealing that the synthesized silver nanoparticles are composed of pure crystalline silver and the particle size is approximately $34 \mathrm{~nm}$. The peak corresponding to (111) plane is more intense than the other planes, suggesting that the (111) plane is in the predominant orientation.

\section{FTIR analysis}

To determine the functional groups on banana peel extract and predict their role in the synthesis of silver nanoparticles, FTIR analysis was performed. The band intensities in different regions of the spectrum for the CBPE and AgNPs (before and after reaction with silver nitrate, respectively) were analyzed and are shown in Fig. 4. There was a shift in the following peaks: 3,394-3,388, 2,920-2,918, 2,355-2,351, 1,643-1,641, 771-769, and 426-422 $\mathrm{cm}^{-1}$. The broad and intense absorption peak at around $3,394 \mathrm{~cm}^{-1}$ corresponds to the $\mathrm{O}-\mathrm{H}$ stretching vibrations of phenols and carboxylic acids. The shift from 3,394 to

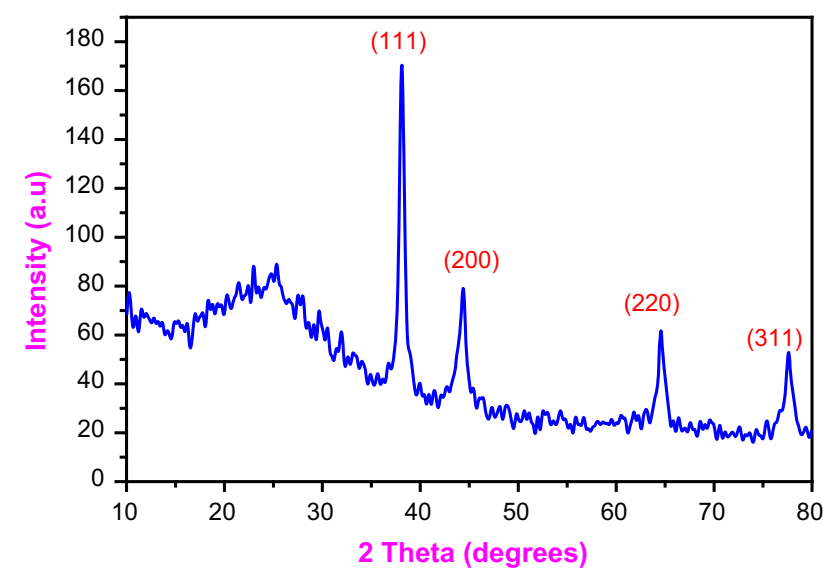

Fig. 3 XRD spectra for the AgNPs prepared with $1 \mathrm{mM}$ aqueous $\mathrm{AgNO}_{3}$ solution with $4 \mathrm{ml} \mathrm{CBPE}$

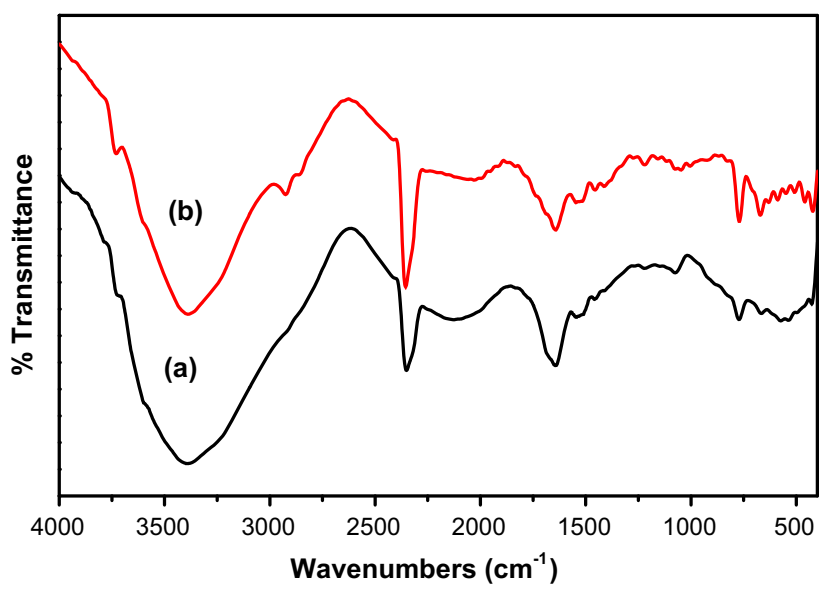

Fig. 4 FT-IR spectra for a CBPE, b Bio-reduced by CBPE silver nanoparticles

$3,388 \mathrm{~cm}^{-1}$ may indicate the involvement of $\mathrm{O}-\mathrm{H}$ functional group in the synthesis of nanoparticles. The peak located at around $2,355 \mathrm{~cm}^{-1}$ was attributed to the $\mathrm{N}-\mathrm{H}$ stretching or the $\mathrm{C}=\mathrm{O}$ stretching vibrations. The peak shift from 2,355 to $2,351 \mathrm{~cm}^{-1}$ implicated that these groups may be involved in the process of nanoparticle synthesis. The peak located at $1,641 \mathrm{~cm}^{-1}$ could be assigned to the $\mathrm{C}=\mathrm{O}$ stretching in carboxyl or $\mathrm{C}=\mathrm{N}$ bending in the amide group. A shift in this peak (from 1,641 to $1,643 \mathrm{~cm}^{-1}$ ) indicated the possible involvement of carboxyl or amino groups of the CBPE powder in nanoparticle synthesis. The peak at 771 and $760 \mathrm{~cm}^{-1}$ corresponds to $\mathrm{C}-$ $\mathrm{H}$ stretching of aromatic compounds. The broad peaks around 426 and $422 \mathrm{~cm}^{-1}$ are related to AgNPs bonding with oxygen from hydroxyl groups. Banana peels are mainly composed of pectin, cellulose and hemicelluloses (Happi Emaga et al. 2007) and the functional groups associated with these polymers as well as the proteinaceous matter may be involved in reducing the silver salt to $\mathrm{Ag}^{0}$.

Morphological studies

Atomic force microscopy

The structure morphology and size of the synthesized silver nanoparticles were characterized by the AFM images. Figure 5 shows lateral and 3D AFM image of biofunctionalized AgNPs. The resultant silver nanoparticle images were observed as spherical in shape. The particle size of the AgNPs was found to be $55 \mathrm{~nm}$ and is also used to verify that the silver nanoparticles were more or less homogenous in size.

\section{Microstructural analysis}

The microstructural analysis of the synthesized AgNPS was carried out by FE-SEM analysis. The FE-SEM image 

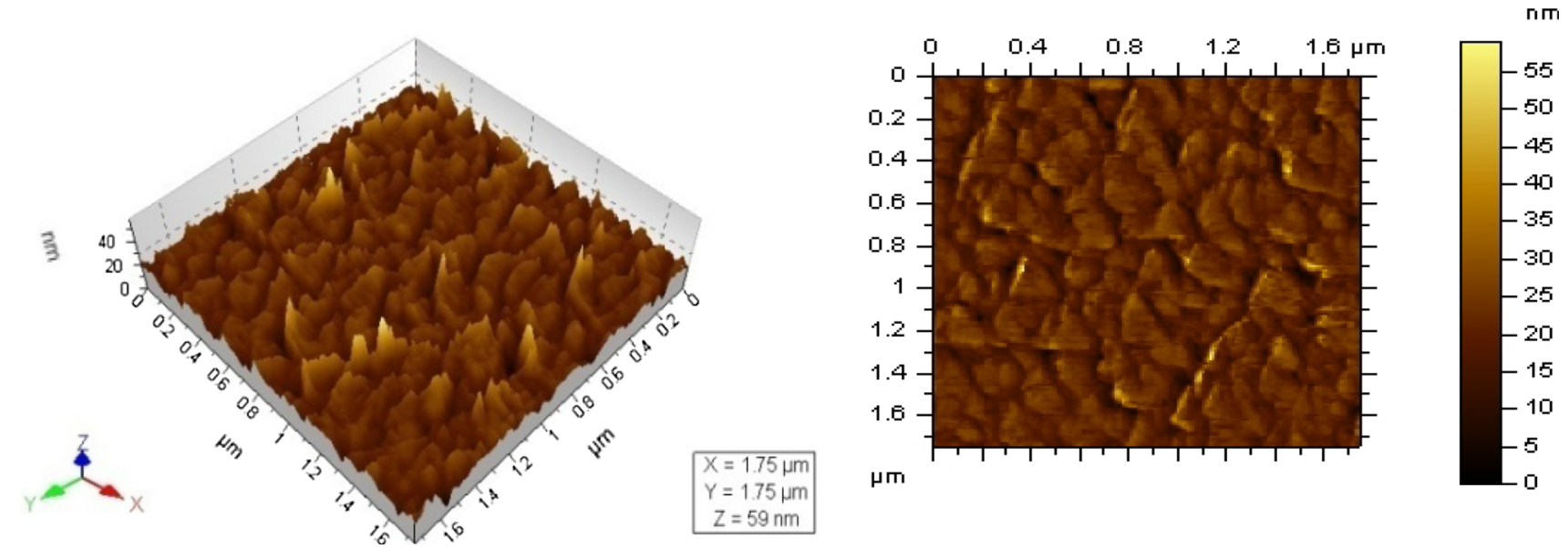

Fig. 5 Atomic force microscopy of AgNPs prepared with $1 \mathrm{mM}$ aqueous $\mathrm{AgNO}_{3}$ solution with $4 \mathrm{ml} \mathrm{CBPE}$

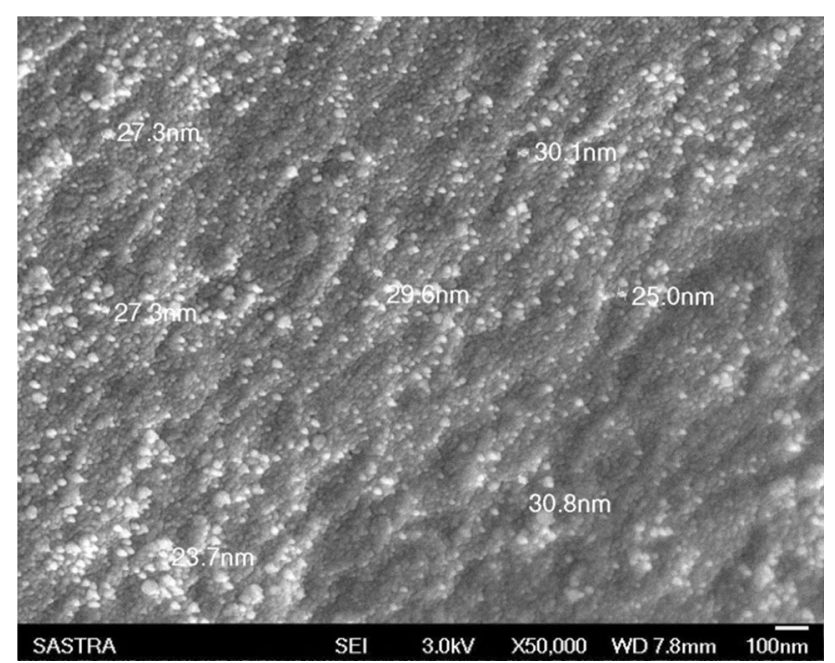

Fig. 6 FESEM for AgNPs prepared with $1 \mathrm{mM}$ aqueous $\mathrm{AgNO}_{3}$ solution with $4 \mathrm{ml} \mathrm{CBPE}$

of the AgNPS is shown in Fig. 6. From the figures, it is observed that all the particles show almost spherical morphology and the diameter of AgNPs is in the range of $23-30 \mathrm{~nm}$. It is good in agreement with XRD and AFM.

\section{DLS measurements}

The DLS instrument is known to measure the shell thickness of a capping or stabilizing agent enveloping the metallic particles along with the actual size of the metallic core. The size distribution vs intensity graph is shown in Fig. 7. The average size of the AgNPs was $297 \mathrm{~nm}$. The large size of particles observed by DLS is due to the fact that the measured size also includes the bio-organic compounds enveloping the core of the AgNPs. Prathna et al. (2011) found the particle size of silver nanoparticles in the order of techniques DLS $>$ AFM $>$ XRD. Moreover, the large particle size could also be due to the various forces of interaction in the solution like van der Waals forces.

The differences in the particle sizes obtained using the microscopic techniques were due to differences in sample preparation and also due to the polydispersity of the sample (as determined by DLS). Different techniques give different size averages, depending on the instrument response to particle numbers, volume, mass or optical property. But this is usually not the case for bio-based synthesis process which produces polydisperse particles. Therefore, a great degree of caution needs to be taken when the particle sizes are measured by various techniques for a bio-based process and needs to be compared to determine their reactivity for specific applications (Kasthuri et al. 2009).

Zeta potential (ZP)

Zeta potential values reveal information regarding the surface charge and stability of the synthesized silver nanoparticles. The zeta potential value of silver nanoparticles obtained from Banana peel extract is $-11 \mathrm{mV}$ (Fig. 7), indicating the stability of the synthesized nanoparticles.

Antibacterial activity of silver nanoparticles

The biologically synthesized silver nanoparticles showed excellent antimicrobial activity against clinically isolated Multidrug-resistant human pathogens such as Gram-positive bacteria viz., Staphylococcus aureus, B. subtilis and Gram-negative bacteria viz., K.pneumonia and E.coli (Fig. 8). The mean inhibitory zone of diameter was measured and tabulated (Table 1). The Gram-negative bacteria $E$. coli and $K$. pneumonia showed maximum zone of inhibition at $10.75 \mathrm{~mm}$ which may be due to the cell wall of Gram-positive bacteria composed of a thick 

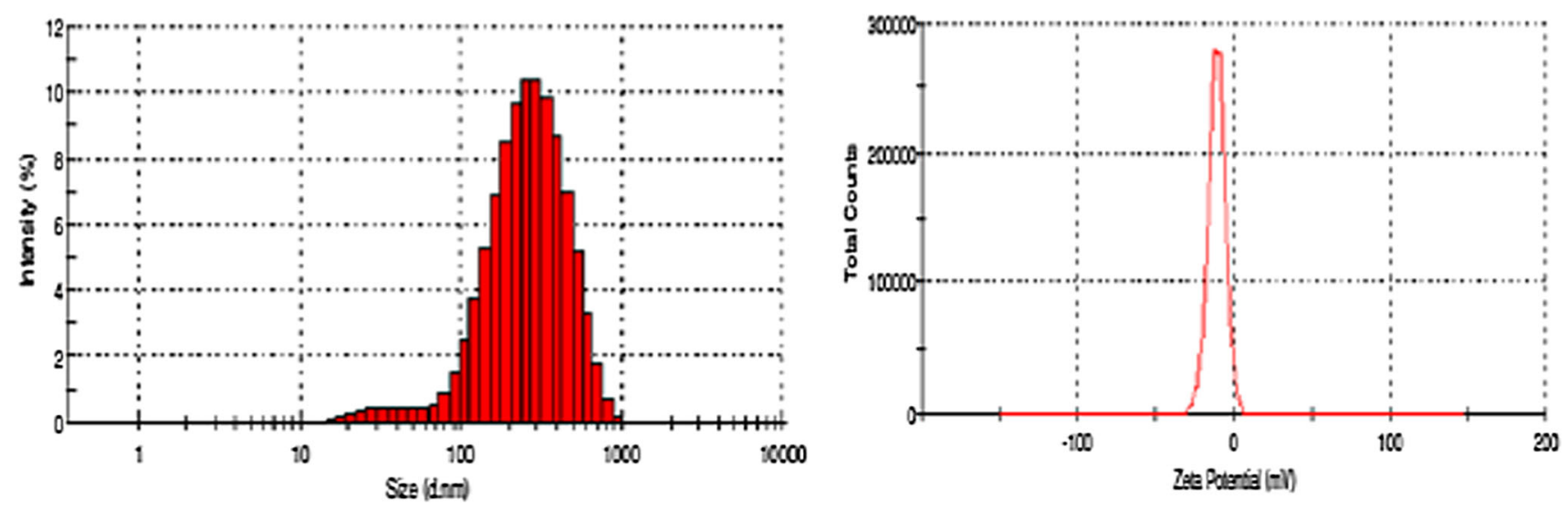

Fig. 7 DLS and Zeta potential for AgNPs prepared with $1 \mathrm{mM}$ aqueous $\mathrm{AgNO}_{3}$ solution with $4 \mathrm{ml} \mathrm{CBPE}$

Fig. 8 Plates showing antibacterial activities of Cavendish banana peel extract (CBPE) and Silver nanoparticles (AgNPs)

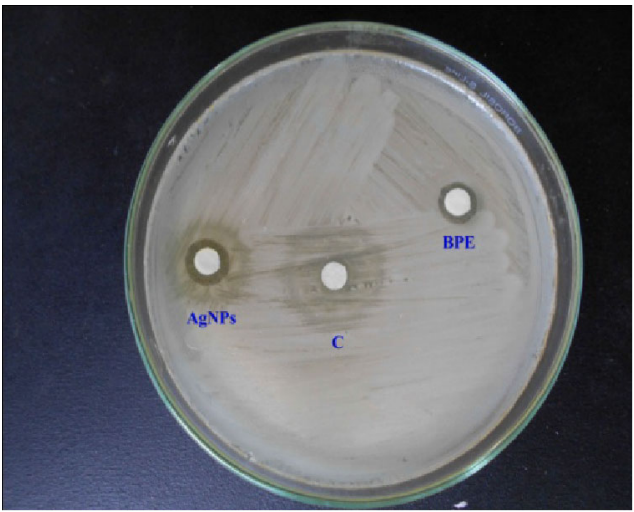

Klebsiella pneumonia

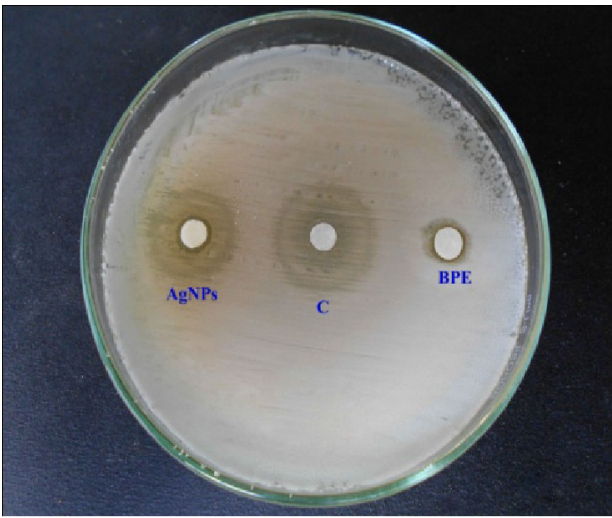

Staphylococcus aureus

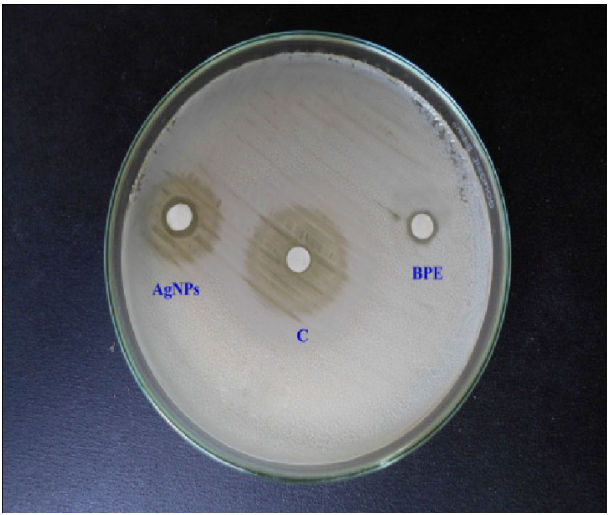

Escherichia coli

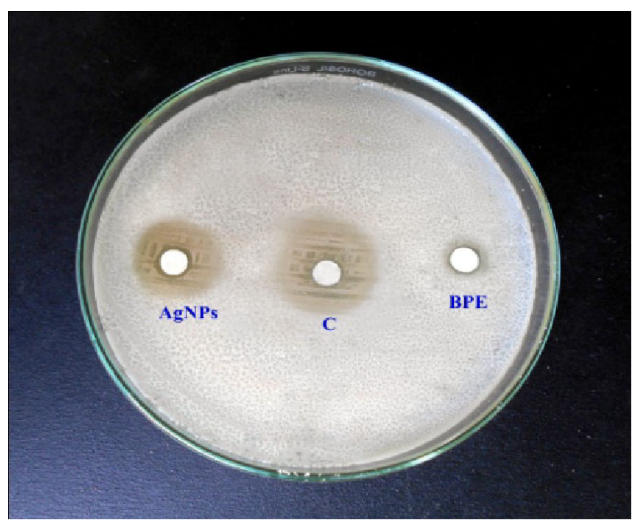

Bacillus subtilis peptidoglycan layer, which consists of linear polysaccharide chains cross linked by short peptides, thus forming more rigid structure leading to difficult penetration of the silver nanoparticle compared to the Gram-negative bacteria where the cell wall possesses thinner peptidoglycan layer (Shrivastava et al. 2007). The high bactericidal activity is certainly due to the silver cations released from Ag nanoparticles that act as reservoirs for the $\mathrm{Ag}^{+}$bactericidal agent. Big changes in the membrane structure of bacteria as a result of the interaction with silver cations lead to the increased membrane permeability of the bacteria (Dibrov et al. 2002; Sondi and Salopek-Sondi 2004). 
Table 1 Inhibitory action of CBPE and Ag-NPs against human pathogenic bacteria

\begin{tabular}{llll}
\hline Plate & Culture & BPE & AgNPs \\
\hline 1 & Klebsiella pneumonia & 13 & 22 \\
2 & Escherichia coli & 12 & 20 \\
3 & Staphylococcus aureus & 11 & 19 \\
4 & Bacillus subtilis & 09 & 17 \\
\hline
\end{tabular}

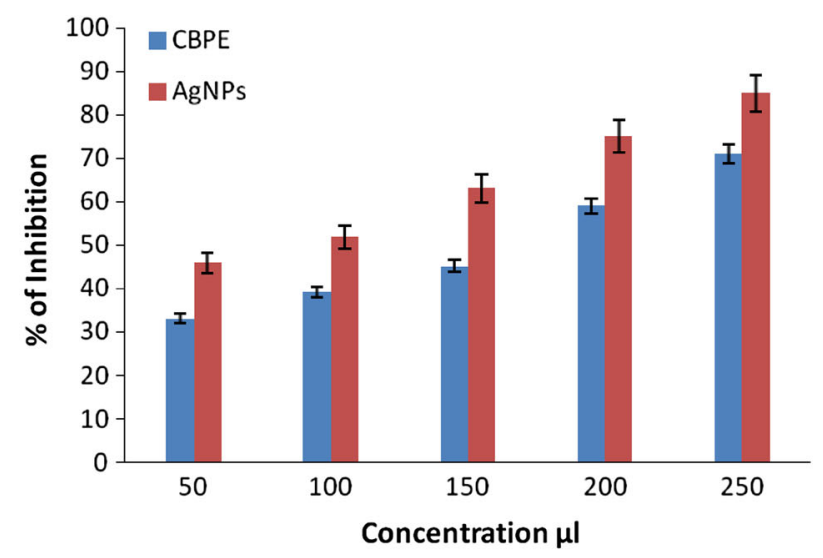

(i)

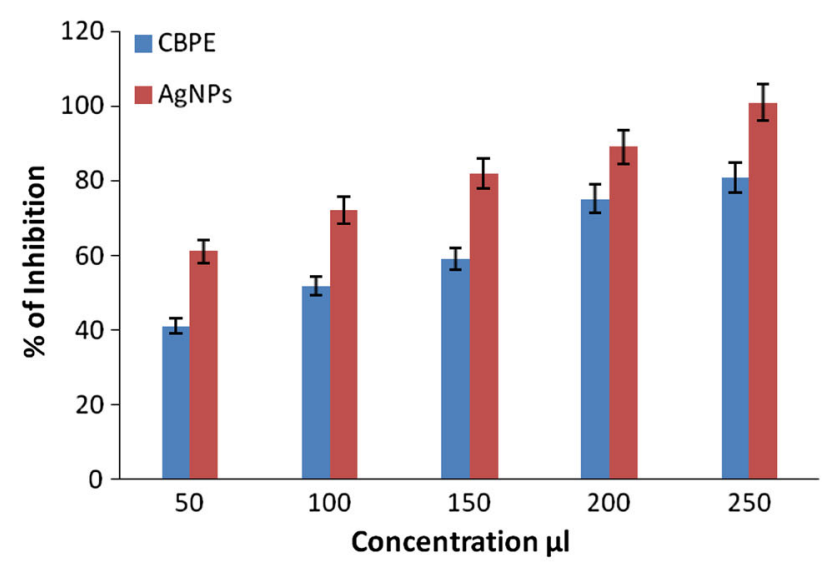

(ii)

Fig. 9 i DPPH free radical scavenging assay for banana peel extract and AgNPs. ii ABTS free radical scavenging assay for banana peel extract and AgNPs

Free radical scavenging activity

\section{DPPH (1,1-Diphenyl-2-Picryl Hydroxyl) radical scavenging assay}

The bar diagram shown in Fig. 9i indicates DPPH radical scavenging potential of pure banana peel extract and synthesized silver nanoparticles mediated by CBPE. The results obtained in the DPPH assay showed effective free radical inhibition by biosynthesized AgNPs. The average percentage inhibition of synthesized AgNPs was $64 \%$ as compared to that of CBPE (49\%) at different concentrations used in this study and the activity increased with increasing concentrations of AgNPs. DPPH radicals react with suitable reducing agents, during which the electrons become paired off and the solution loses color stoichiometrically depending on the number of electrons taken up (Subhasree et al. 2009). In this experiment, the solution progressively reduced to a yellow colored product, diphenylpicryl hydrazine, with the addition of the extracts in a concentration-dependent manner.

\section{ABTS [2,2'-Azinobis-(3-Ethylbenzothiazoline-6-Sulfonic Acid)] Assay}

Biosynthesized nanoparticles show considerable potential in in vitro trials for free radical scavenging activity. Both banana peel extract and Ag nanoparticles have potential for scavenging ABTS radical by surface reaction phenomenon in a dose-dependent manner shown in Fig. 9ii. This study also demonstrates the high sensitivity of nanoparticles against ABTS radical as compared to peel extract. ABTS scavenging percentages of the nanoparticles increased from $42.47 \%$ for peel extract and $84.71 \%$ in case of AgNPs. The mechanism involved in this free radical scavenging feature of nanoparticles has significant impact in the sphere of nanomedicine.

\section{Conclusion}

Silver nanoparticles were produced by the direct interaction of silver nitrate with peel extract in aqueous media without the intervention of any external man-made chemicals. Therefore, this reaction pathway satisfies all the conditions of a $100 \%$ green chemical process. The amount of plant material is found to play a critical role in controlling the size and size dispersity of nanoparticles in such a way that smaller silver nanoparticles and narrow size distribution are produced when more CBPE is added in the reaction medium. The methodology employed here is very simple, easy to perform, inexpensive, and eco-friendly. The colloidal solutions are stable, suggesting that CBPE can be used as both reducing and stabilizing agent for the preparation of $\mathrm{Ag}$ nanoparticles.

Acknowledgments The authors gratefully acknowledge the DSTINSPIRE sponsored programme, Department of Science and Technology, New Delhi, India for funding the research development (Ref no IF 130264) to carry out this work. 
Open Access This article is distributed under the terms of the Creative Commons Attribution License which permits any use, distribution, and reproduction in any medium, provided the original author(s) and the source are credited.

\section{References}

Ahmad N, Sharma S, Rai (2012) Rapid green synthesis of silver and gold nanoparticles using peels of Punica granatum. Int $\mathrm{J}$ Nanopart 5:185-195

Albrecht MA, Evan CW, Raston CL (2006) Green chemistry and the health implications of nanoparticles. Green Chem 8:417-432

Annadurai G, Juang RS, Lee DJ (2003) Adsorption of heavy metals from water using banana and orange peels. Water Sci Technol 47:185-190

Arawande JO, Komolafe EA (2010) antioxidative potentials of banana and plantain peel extracts on crude palm oil. Ethnobot Leafl 14:559-569

Bar H, Bhui DK, Sahoo GP, Sarkar P, Pyne S, Misra A (2009) Green syntheis of silver nanoparticles using seed extract of Jatropha curcas. Colloids Surf A Physic- ochem Eng Asp 348:212-216

Begum NA, Mondal S, Basu S, Laskar RA, Mandal D (2009) Biogenic synthesis of $\mathrm{Au}$ and $\mathrm{Ag}$ nanoparticles using aqueous solution of black tea leaf extracts. Colloids Surf B Biointerfaces 7(1):113-118

Bharani M, Karpagam T, Varalakshmi B, Gayathiri G, Priya LK (2012) Synthesis and characterization of silver nano particles from Wrightia tinctoria. Int J Appl Biol Pharm Technol 3:58-63

Brand-Williams W, Cuvelier ME, Berset C (1995) Use of Free Radical Method to Evaluate Antioxidant Activ-ity. Lebensmittel Wissenschaft und Technologie 28:25-30

Dibrov P, Dzioba J, Gosink KK, Hase CC (2002) Chemiosmotic Mechanism of Antimicrobial Activity of $\mathrm{Ag}^{+}$in Vibrio cholerae Antimicrob. Agents Chemother 46:2668-2670

Easu SR, Roberto SB, Ocotlan-Flores J, Saniger JM (2010) Synthesis of AgNPs by sonochemical induced reduction application in SERS. J Nanopart Res 9:77

Essien JP, Akpan EJ, Essien EP (2005) Studies on mould growth and biomass production using waste banana peel. Bioresour Technol 96:1451-1456

Ganesh Babu MM, Gunasekaran P (2009) Production and structural characterization of crystalline silver nanoparticles from Bacillus cereus isolate. Colloids Surf B Biointerface 74:191-195

Gong P, Li H, He X, Wang K, Hu J, Tan W (2007) Preparation and antibacterial activity of $\mathrm{Fe}_{3} \mathrm{O}_{4}$ and $\mathrm{Ag}$ nanoparticles. Nanotechnol 18:604-611

Guangquan L, Dan H, Yongqing Q, Buyuan G, Song G, Yan C (2012) Fungus mediated green synthesis of silver nanoparticles using Aspergillus terreus. Int J Mol Sci 13:466-476

Happi Emaga T, Robert C, Ronkart SN, Wathelet B, Paquot M (2007) Dietary fibre components and pectin chemical features of peels during ripening in banana and plantain varieties. Bioresour Technol 99:4346-4354

He R, Qian X, Yin J et al (2002) Preparation of polychrome silver nanoparticles in different solvents. J Mater Chem 12:3783-3786

Jeong SH, Yeo SY, Yi SC (2005) The effect of filler particle size on the antibacterial properties of compounded polymer/silver fibers. J Mat Sci 40:5407-5411

Kanazawa K, Sakakibara H (2000) High Content of Do- pamine, a Strong Antioxidant in Cavendish Banana. J Agric Food Chem 48(3):844-848
Kasthuri J, Veerapandian S, Rajendiran N (2009) Biological synthesis of silver and gold nanoparticles using apiin as reducing agent. Colloid Surf B Biointerf 68:55-60

Kaviya S, Santhanalakshmi J, Viswanathan B, Muthumar J, Srinivasan K (2011) Biosynthesis of silver nanoparticles using Citrus sinensis peel extract and its antibacterial activity. Spectrochim Acta Part A Mol Biomol Spectrosc 7:594-598

Martinez-Castanon GA, Nino-Martinez N, Martinez-Gutierrez F, Martinez- Mendoza JR (2008) Synthesis and antibacterial activity of silver nanoparticles with different sizes. J Nanopart Res 10:1343-1348

Navaladian S, Viswanathan B, Viswanath RP, Varadarajan TK (2007) Thermal decomposition as route for silver nanoparticles. Nanoscale Res Lett 2:44-48

Nidhi N, Santosh K, Ghosh T, Dutta PK (2009) Preparation of Chitosan based silver nano composites by a facile method. International Conference on Optics and Photonics, Chandigarh: CSIO

Novak JP, Feldheim DL (2000) Assembly of phenylacetylene bridged silver and gold nanoparticle arrays. J Am Chem Soc 122:3979-3980

Osma JF, Toca Herrera JL, Rodriguez Couto R (2007) Banana skin: a novel waste for laccase production by Trametes pubescens under solid-state conditions. Application to synthetic dye decolouration. Dyes Pigments 75:32-37

Padma S, Shukla D (2012) Biosynthesis of silver nanoparticles using lemon leaves extract and its application for antimicrobial finish on fabric. Appl Nanosci 2:163-168

Parmar HS, Kar A (2008) Medicinal values of fruit peels from Citrus sinensis, Punica granatum, and Musa paradisiaca with respect to alterations in tissue lipid per- oxidation and serum concentration of glucose, insulin, and thyroid hormones. J Med Food 11:376-381

Phanjom P, Zoremi E, Mazumder J, Saha M, Baruah SB (2012) Green synthesis of silver nanoparticles using leaf extract of Myrica esculenta. Int J NanoSci Nanotechnol 3:73-79

Prathna TC, Chandrasekaran N, Raichur M, Mukherjee A (2011) Biomimetic synthesis of silver nanoparticles by Citrus limon (lemon) aqueous extract and theoretical prediction of particle size. Colloid Surf B Biointerf 82:152-159

Re R, Pellegrini N, Proteggente A, Pannala A, Yang M, Rice-Evans C (1999) Antioxidant activity applying improved ABTS radical cation decolorization assay. Free Radic Biol Med 26(9-10):1231-1237

Shanmugavadivu M, Selvam Kuppusamy, Ranjithkumar R (2014) Synthesis of pomegranate peel extract mediated silver nanoparticles and its antibacterial activity. Am J Adv Drug Deliv 2(2):174-182

Shrivastava S, Bera T, Roy A, Singh G, Ramachandrarao P, Dash D (2007) Characterization of enhanced antibacterial effects of novel silver nanoparticles. Nanotechnology 18:225103-225111

Someya S, Yoshiki Y, Okubo K (2002) Antioxidant compounds from bananas (Musa cavendish). Food Chem 79(3):351-354

Sondi I, Salopek-Sondi B (2004) Silver nanoparticles as antimicrobial agent: a case study on E. coli as a model for Gram-negative bacteria. J Colloid Interface Sci 275:177-182

Sreeram KJ, Nidin M, Nair BU (2008) Microwave assisted template synthesis of silver nanoparticles. Bull Mater Sci 31(7):937-942

Starowicz M, Stypula B, Banas J (2006) Electrochemical synthesis of silver nanoparticles. Electrochem Commun 8(2):227-230

Subhasree B, Baskar R, Laxmi Keerthana R, Susan R L, Rajasekaran $P$ (2009) Evaluation of antioxidant potential in selected green leafy vegetables. Food Chem 115(4):1213-1220 
Sun L, Wang L, Song Yonghai, Guo C, Sun Y, Peng C (2008) Aggregation- based growth of silver nanowires at room temperature. App Surf Sci 254:2581-2587

Tewari HK, Marwaha SS, Rupal K (1986) Ethanol from banana peels. Agric Wastes 16:135-146
Yang Ning, Li Wei-Hong (2013) Mango peel extract mediated novel route for synthesis of silver nanoparticles and antibacterial application of silver nanoparticles loaded onto non-woven fabrics. Ind Crops Prod 48:81-88 